\title{
L'impact de la « participation musulmane » sur le mouvement altermondialiste en Grande-Bretagne et en France
}

Timothy Peace

\section{(2) OpenEdition \\ Journals}

Édition électronique

URL : http://journals.openedition.org/conflits/13213

DOI : $10.4000 /$ conflits. 13213

ISSN : 1777-5345

Éditeur :

CCLS - Centre d'études sur les conflits lilberté et sécurité, L'Harmattan

Édition imprimée

Date de publication : 20 juillet 2008

Pagination : 109-128

ISBN : 978-2-296-05909-2

ISSN : 1157-996X

Référence électronique

Timothy Peace, «L'impact de la « participation musulmane » sur le mouvement altermondialiste en Grande-Bretagne et en France », Cultures \& Conflits [En ligne], 70 | été 2008, mis en ligne le 04 janvier 2010, consulté le 30 mars 2021. URL : http://journals.openedition.org/conflits/13213 ; DOI : https:// doi.org/10.4000/conflits.13213 


\title{
L'impact de la "participation musulmane " sur le mouvement altermondialiste en Grande-Bretagne et en France
}

\author{
Timothy PEACE
}

Timothy Peace est doctorant au département de sciences politiques et sociales de l'Institut universitaire européen de Florence. Ses recherches portent sur la participation des musulmans européens dans les mouvements sociaux et sur la relation entre religion et politique en Europe. Email : timothy.peace@eui.eu

$\mathrm{D}$ epuis 2001, en France comme en Grande-Bretagne, les « musulmans 2 » et les associations qui les représentent ont été amenés à participer à plusieurs occasions au mouvement altermondialiste ${ }^{3}$. On a notamment pu le constater lors des Forum sociaux européens (FSE) qui se sont tenus dans les capitales de ces deux pays ${ }^{4}$. Cet engagement s'étant récemment affaibli, il est possible d'y voir un cas d'" altermondialisme oublié ». On a souvent pointé ces pays en raison de leur attitude divergente vis-à-vis de leurs minorités respectives et sur la

1. Une version antérieure de cet article a été présentée au colloque «Action collective et exclusion sociale en Europe » à l'ENS-LSH de Lyon, 11 janvier 2008. Nous voudrions remercier les deux évaluateurs extérieurs pour leurs commentaires et suggestions ainsi que Alexandre Diaz pour son aide à la traduction. Nous sommes aussi reconnaissant envers tous les militants qui ont accepté de nous parler de leurs expériences.

2 . Le terme «musulman » est utilisé pour décrire les acteurs qui, dans l'ensemble, se sont mobilisés au sein de la société civile, sur la base de leur confession religieuse, ou qui ont intégré des associations confessionelles musulmanes. Cela ne dénote pas les pratiques individuelles de cette foi et je suis conscient du problème que peut causer ce terme. Néanmoins, nous préférons celui-ci à d'autres terminologies comme « issus de l'immigration » car cela a tendance à détourner l'attention du fait que ces acteurs sont des citoyens français et britanniques et que certains ne sont même pas d'origine immigré. De plus, c'était la nature «islamique » de certains groupes qui a été la source de nombreuses tensions que nous décrirons plus loin.

3. Certains utilisent l'expression « mouvement pour une justice globale » pour la dynamique en Grande-Bretagne. Voir Rootes C., Saunders C., «Le développement du mouvement pour une justice globale en Grande-Bretagne » in Sommier I., Fillieule O., Agrikoliansky E., (dirs.) Généalogie des mouvements altermondialistes en Europe, Paris, Karthala, 2008, pp. 67-85.

4. Pour mémoire : Le $1^{\text {er }}$ Forum social européen (FSE) s'est tenu à la forteresse de Basso de Florence en Italie du 7 au 10 novembre 2002. Le $2^{\mathrm{e}}$ Forum social européen s'est tenu à Paris, Saint-Denis, Bobigny et Ivry-sur-Seine du 12 au 15 novembre 2003. Le $3^{\mathrm{e}}$ Forum social européen s'est déroulé à Londres du 14 au 17 octobre 2004. Le 4e Forum social européen s'est déroulé les 4, 5, 6, et 7 Mai 2006, à Athènes. 
place qu'y occupe la religion dans la sphère publique. Ces deux cas offrent donc aux chercheurs l'opportunité d'étudier comment ces différentes conceptions affectent les mouvements sociaux et leurs participants. Nous souhaitons montrer dans cet article que l'élément le plus notable de cette mobilisation musulmane est l'impact de celle-ci sur des mouvements altermondialistes eux-mêmes et la manière dont elle a remis en question leur propre représentation de mouvements ouverts et «tolérants». De nombreux spécialistes des mouvements sociaux ont remarqué le fait que les acteurs de ces mouvements doivent souvent faire face à des dilemmes causés par le poids des identités religieuses et la difficulté de faire coexister celles-ci avec d'autres critères d'identification ${ }^{5}$. Cet article cherche à mettre ces difficultés en avant, ainsi que les limites de la supposée «tolérance » des acteurs «traditionnels » de l'altermondialisme, confrontés à la participation des musulmans. Les FSE servent de lieu de rencontre et de discussion pour une myriade de groupes se réclamant de la mouvance altermondialiste. Cependant, l'événement en lui-même consiste à la fois en un forum officiel et en un « espace alternatif / autonome » agissant comme un événement annexe, composé de tous les groupes qui rejettent le processus officiel d'organisation. A l'intérieur du mouvement lui-même, on fait souvent référence à ce clivage en usant de l'expression "borizontaux contre verticaux», chacun des groupes ayant des conceptions opposées de la politique, des enjeux du FSE et du mouvement en général ${ }^{6}$. Nous étudierons ici uniquement les forums officiels - en particulier ceux qui se sont tenus en région parisienne en 2003 et à Londres en 2004 - et nous ne traiterons donc que des acteurs « verticaux».

\section{Expliquer la participation musulmane dans l'altermondialisme et le processus du FSE}

Les implications des associations musulmanes et leurs militants dans ce processus sont extrêmement différentes d'un pays étudié à l'autre. En GrandeBretagne, l'opposition aux opérations de guerre menées en Afghanistan et en Irak, principalement regroupée au sien de la Stop the War Coalition (SWC), a joué un rôle majeur dans la mobilisation musulmane et a créé les conditions d'un rapprochement avec des altermondialistes. En France, la mobilisation musulmane est davantage à rapprocher d'activités et d'associations actives durant de longues années dans les banlieues.

\section{La mobilisation britannique. Viser les communautés}

De nombreux observateurs extérieurs ont tendance à voir dans le 11 septembre 2001 et ses conséquences le point de départ de la mobilisation des

5. Della Porta D., Diani M., Social movements: An introduction (2e ed.), Malden, Blackwell, 2006, p. 100.

6. Voir Flesher Fominaya C., "Autonomous movements and the institutional left: Two approaches in Tension in Madrid's anti-globalization network", South European Society E Politics, septembre 2007 , n²12, pp. 335-358. 
musulmans britanniques autour des thèmes chers aux altermondialistes. Pourtant, il existait déjà un groupe de musulmans issus de classes moyennes, basé à Londres, qui organisait des débats autour de sujets comme la mondialisation économique et les changements climatiques, dans le cadre d'une association appelée The City Circle ${ }^{7}$. A la suite du 11 septembre 2001, un groupe anti-guerre s'est formé dans les rangs de cette association et a pris le nom de JustPeace (JP), lequel a rejoint quelques temps plus tard le comité de pilotage de la SWC ${ }^{8}$. Les musulmans britanniques étaient en très grande majorité opposés à la campagne de bombardement contre les Talibans en Afghanistan en octobre 2001. La tentative du gouvernement britannique de convaincre l'opinion musulmane de la nécessité des bombardements était condamnée à l'échec, sachant que le Muslim Council of Britain (MCB) ${ }^{9}$ refusait de soutenir de telles opérations militaires. Néanmoins, dans le même temps, la réticence initiale du MCB à s'opposer officiellement aux actions militaires et à diriger l'opposition musulmane a ouvert un espace pour des groupes comme JP. Leurs principales tâches consistaient à préparer et à distribuer des tracts de la SWC, à rassembler des personnes pour participer aux manifestations, à créer des bannières et des affiches, à dispenser des formations relatives aux techniques d'expression à utiliser face aux médias et à mettre en place des espaces dédiés à la prière. On a donc assisté à l'émergence d'un nouvel aspect de la mobilisation, celui d'une «volonté des plus jeunes musulmans à former des alliances opportunes avec des groupes en debors de leur communauté, ce qui n'était certainement pas le cas pendant l'affaire Rushdie en 1989 et la guerre du Golfe en 199110 ». Ces jeunes activistes ont aussi contribué à mobiliser d'anciens membres de leur communauté et ont souvent ouvré à interpeler les consciences dans les mosquées.

La SWC est créée le 21 septembre 2001. Sa direction est constituée de diverses personnalités de la gauche radicale britannique, en particulier dominée par le Socialist Workers Party (SWP). Ce dernier a joué un rôle essentiel dans la mouvance altermondialiste britannique et a été présent à tous les FSE, souvent sous la bannière de ses organisations satellites telles que Globalise Resistance ${ }^{11}$ ou Project $K^{12}$. On peut y voir un moyen de contourner l'interdiction semi-officielle de participation frappant les partis politiques. La SWC

7. Formé en 1999 en tant que forum de discussion sur des sujets d'actualité pour les musulmans britanniques, voir : http://www.thecitycircle.com

8. Le but de JP était de promouvoir «la participation musulmane dans les mouvements qui font campagne pour la liberté face à l'oppression et à l'injustice ». Ses membres participaient à de nombreux séminaires lors des deuxième et troisième Forums sociaux européens, mais ils n'étaient pas réellement impliqués dans leur organisation.

9. Fédération d'organisations musulmanes qui représente la communauté musulmane britannique auprès du gouvernement. Sur le MCB et son dilemme sur l'Afghanistan, voir Birt J., "Lobbying and marching: British Muslims and the State", in Abbas T., (ed.), Muslim Britain: Communities under Pressure, London, Zed books, 2005, pp. 92-106.

10. Ibid., p. 102.

11. Voir le site : http://www.resist.org.uk/

12. Voir le site : http://www.projet-k.org/ 
est très favorable à la participation musulmane; les trotskistes du SWP ont atténué leurs assertions habituelles concernant l'importance du socialisme et du marxisme afin de maintenir un front uni et d'encourager les musulmans à se joindre à la coalition. Outre "Stop the War", ils ont adopté deux nouveaux slogans pour les manifestations dont le but était de montrer leur soutien à la communauté musulmane: "Defend civil liberties" et "Resist the racist backlash". Ghayasuddin Siddiqui du Muslim Parliament (MP) et Asad Rehman du groupe anti-raciste Newham Monitoring Project (NMP) ont également rejoint la coalition dès ses débuts ${ }^{13}$. Selon les leaders de la SWC, des groupes comme le NMP « ont aidé à mobiliser les communautés noire et asiatique et à faire de l'anti-racisme un élément central de la campagne 14 ». Cette façon de faire, propre à la Grande-Bretagne, consistant à cibler des « communautés » correspond au concept de frame bridging développé par David Snow, Burke Rochford, Steven Worden et Robert Benford et contraste clairement avec celle adoptée en France ${ }^{15}$. Salma Yaqoob, une autre figure de proue musulmane de cette coalition, a contribué à l'implantation de la SWC à Birmingham et, quelques temps plus tard, elle est devenue conseillère municipale de la ville pour le parti politique Respect (voir infra). Au printemps 2002, la SWC s'allie avec certains membres de la Muslim Association of Britain (MAB) : Azam Tamimi, Mohammed Sarwala et Anas Altikriti 16. Ce n'est pas la question de l'Afghanistan ou la possible invasion de l'Irak qui a rassemblé ces groupes, mais la cause palestinienne. La MAB avait précédemment réussi à organiser des manifestations de solidarité pour la Palestine et, lorsque la SWC et la MAB se sont rendues compte qu'elles avaient toutes deux planifié un défilé en septembre 2002, elles ont entamé des négociations pour y présenter un front uni. A l'instar de la Campaign for Nuclear Disarmament (CND), un mouvement pacifiste britannique établi un certain nombre d'années, la MAB est ainsi devenue un partenaire important de la SWC. Mais ce partenariat est quelque peu surprenant au regard des nombreuses réserves émises par la gauche radicale et la forte opposition interne au MAB.

13. MP est une petite association, dirigée par Kalim Siddiqui de 1992 jusqu’à sa mort en 1996. Elle est désormais dirigée par le $\mathrm{D}^{\mathrm{r}}$ Siddiqui. Le NMP fut créé en 1980 pour mettre en place des campagnes anti-racistes et pour suivre de près les réactions de la police vis-à-vis des crimes racistes. La plupart de ses membres appartiennent à la «communauté noire » plutôt que musulmane même si Asad Rehman (de confession musulmane) en était le représentant aux réunions préparatoires au FSE.

14. Murray A., German L., Stop the War: the Story of Britain's Biggest Mass Movement, London, Bookmarks, 2005, p. 49. Le terme " asiatique » («asian») se réfère aux personnes dont les familles sont originaires du sous-continent indien. La plupart des musulmans de GrandeBretagne sont donc des « asiatiques ».

15. Snow D., Rochford Burke E., Worden SK., Benford R., "Frame Alignment Process, Micromobilization, and Movement Participation”, American Sociological Review, août 1986, n०51, pp. 464-481.

16. La MAB, membre du MCB, est en grande partie composée de musulmans appartenant à la communauté arabe. Créée en 1997, elle est le résultat d'une scission au sein de l'Islamic Society of Britain (ISB), une partie de ses membres ayant voulu renforcer des liens avec les Frères musulmans en Egypte. 
La participation des musulmans de Grande-Bretagne aux FSE est donc en grande partie le résultat du mouvement anti-guerre, la collaboration avec des mouvements anticapitalistes ayant permis l'échange et le développement d'idées, ainsi que l'introduction d'autres activités au sein de la mouvance altermondialiste. Ainsi, le groupe JP a été invité au deuxième FSE à Paris et a participé à l'animation de séminaires. Mais c'est naturellement le troisième FSE à Londres en 2004 qui a attiré la plus grande mobilisation de musulmans britanniques. Cela ne s'explique pas seulement par la proximité géographique et le succès du mouvement anti-guerre, mais aussi par le désir du comité organisateur d'être pionnier dans ce qu'il considérait comme les « questions musulmanes » (et, d'une certaine façon, de les exploiter), et notamment celle du foulard qui a été banni des écoles publiques françaises quelques mois auparavant. Le SWP ainsi qu'un petit groupe de personnes travaillant pour le maire de Londres, Ken Livingstone, au sein de la Greater London Authority (GLA) ${ }^{17}$ sont les principaux organisateurs du FSE de Londres. Livingstone, fortement opposé à la « loi du 15 mars 200418 », a accueilli une conférence en juillet 2004 organisée par l'Assemblée pour la protection de l'Hijab 19. Le maire souhaitait aussi s'assurer que le FSE reflète bien « les diverses communautés de Londres » et qu'il promeuve l'image multiculturelle de la capitale. Le séminaire intitulé « Hijab : le droit de la femme de choisir » qui s'est tenu lors de ce forum visait clairement à condamner la nouvelle loi française et a généré de nombreuses tensions avec les délégués français présents soutenant cette loi. Ce séminaire, comme de nombreux autres, a été organisé par la National Assembly Against Racism (NAAR), une association gérée par Lee Jasper, le conseiller principal à la Politique des égalités de Livingstone. Le maire devait prononcer un discours sur l'antifascisme en une séance plénière, mais il s'est ravisé devant les risques d'interruption des militants 20.

La présence musulmane lors du troisième FSE a également constitué une mobilisation populaire. Des groupes comme l'International Muslim Activist Network (IMAN) se sont créés pour le FSE dans le but de mettre en relation les musulmans et les militants de la mouvance altermondialiste. La London Muslim Coalition (LMC) ${ }^{21}$ a participé à l'organisation de nombreux séminaires et événements, bientôt rejointe par une série d'autres petits groupes de

17. Celui-ci était composé de figures comme John Ross, Redmond O’Neill, Neale Coleman et Lee Jasper, tous d'importants assistants et conseillers de Ken Livingstone. Les deux premiers sont membres du Socialist Action, un groupe séparatiste de l'International Marxist Group. Le rôle de ces individus a récemment suscité l'intérêt des médias en Grande-Bretagne. Oliver J., "Ken's aides in secret Marxist cell”, Sunday Times, 20 janvier 2008.

18. Loi n²004-228 du 15 mars 2004 encadrant, en application du principe de laïcité, le port de signes ou de tenues manifestant une appartenance religieuse dans les écoles, collèges et lycées publics.

19. Une campagne initiée par la Muslim Women Society et la MAB. Voir http://www.prohijab.net

20. Environ 200 personnes ont envahi la scène pour dénoncer l'organisation du FSE et ont brandi des bannières aux slogans comme "Ken's party, war party" et "ESF Another world is for sale".

21. La LMC a été créée et dirigée par Kumar Murshid, un ancien conseiller municipal du parti travailliste qui quitta ce parti en 2007 pour rejoindre Respect. Il a également travaillé comme conseiller auprès de Ken Livingstone et a été l'un des membres fondateurs du NAAR. 
Londres comme la Kingston Muslim Women's Welfare Association et le London Pakistan Network. Néanmoins, il est important de souligner qu'un certain nombre de ces groupes étaient principalement venus pour faire de la figuration et ne peuvent donc être considérés comme centraux dans le processus du FSE. De nombreuses associations créées pour défendre les libertés civiles après la législation antiterroriste y étaient également impliquées : l'East London Campaign Against Terrorism (liée au NMP), Stop Police Terror, ou la Campaign Against Criminalising Communities (CAMPACC) où les musulmans étaient représentés en bonne proportion. Au vu de la contribution de tous ces groupes dans l'organisation d'événements lors du forum, on ne s'étonnera pas qu'au dernier moment, et malgré le fait qu'ils n'aient pas pris part à l'organisation du FSE en amont, les organismes nationaux les plus importants comme le MCB, la Federation of Student Islamic Societies (FOSIS) et $Q-N e w s$ (un magazine musulman britannique national) se soient mis à soutenir le FSE et qu'ils aient mis en avant leurs porte-paroles lors des séminaires.

\section{Le contexte français. Les banlieues se mobilisent}

En France, parler de la mobilisation musulmane est plus problématique car, contrairement à la Grande-Bretagne, les groupes impliqués ne se mobilisent pas toujours autour d'une identité islamique. En effet, comme l'expliquent Lucie Bargel, Choukri Hmed et Lilian Mathieu, la manière dont le groupe se désigne lui-même constitue un problème pour celui-ci :

«La définition même du groupe par les acteurs qui prétendent le représenter est, en effet, un objet de luttes afin de l'intégrer dans l'ensemble des populations victimes de la mondialisation néolibérale, tout en le distinguant d'autres catégories (étrangers, jeunes, précaires, etc.) ${ }^{22}$. »

Ainsi les groupes concernés se sont dotés de noms à rallonge comme «mouvements associatifs musulmans et/ou issus des quartiers ou de l'immigration » ou «militants des quartiers populaires, de l'immigration et/ou musulmanes». Ce qui pourrait comporter des risques de confusion est en réalité un indicateur du fait que, bien que de nombreux activistes puissent être de culture musulmane, ils ne se mobilisent pas nécessairement sur la base de leur foi et parfois, ne la pratique pas du tout. Le Mouvement de l'immigration et des banlieues (MIB), un groupe travaillant pour que justice soit rendue aux victimes de bavures policières et contre la discrimination judiciaire dans les banlieues, est un bon exemple. La question de la religion n'est pas pertinente pour leurs activités, bien que, ces dernières années, ils se soient mobilisés sur des problèmes d'ordre religieux, comme l'interdiction du voile ou l'islamophobie. Ils se disent eux-mêmes

22. Bargel L., Hmed C., Mathieu L., "Causes identitaires et groupes enjeux », in Agrikoliansky E., Sommier I. (dirs.), Radiographie du mouvement altermondialiste : le second Forum social européen, Paris, La Dispute, 2005, p. 230. 
représentants (mais pas uniques représentants) des «quartiers » et des cités populaires et « issus de l'immigration » plutôt que musulmans.

Les associations musulmanes qui se sont impliquées dans le processus du FSE étaient le Collectif des musulmans de France (CMF) et le réseau Présence musulmane, toutes deux liées à Tariq Ramadan (voir infra) ${ }^{23}$. A travers la campagne de soutien à Ramadan, le "Comité pour la libre expression des musulmans en France » (suite à l'interdiction qui lui a été faite de pénétrer sur le sol francais fin $1995)^{24}$, les liens existants entre les associations musulmanes et les associations des droits de l'Homme, de solidarité et anti-racistes se sont renforcés ${ }^{25}$, ce qui a mené à la création de la commission « Islam et laïcité 26 » en 1997. Ainsi, les militants des banlieues sont donc entrés en contact avec le monde des altermondialistes, encore à l'état embryonnaire. Ces contacts se sont renforcés dans les années 2000-2001 grâce au travail du MIB qui avait collaboré avec de nombreux groupes et associations qui, plus tard, allaient être les fondateurs du réseau « No Vox 27 ». Le MIB, acteur le plus important des banlieues, a joué un rôle pivot dans la fédération des différentes tendances et a jeté un pont entre les différents groupes. Un autre point de contact a été la Confédération paysanne menée par José Bové qui est, pour beaucoup, le symbole de l'altermondialisme en France. En 2001, José Bové s'est rendu à la première des deux visites de solidarité en Cisjordanie avec une délégation incluant quelques personnes du MIB. Après cette visite, l'idée de lier la lutte palestinienne à la bataille contre la « mondialisation néolibérale » émerge et prend corps ${ }^{28}$. Lors du premier FSE à Florence, ces groupes ont fait leur entrée dans la galaxie de l'altermondialisme. L'influence du nouveau contexte politique post-11septembre sur l'altermondialisme se faisait alors largement sentir, à tel point que l'opposition à la « guerre contre le terrorisme » et au développement des mesures de sécurité est devenue des enjeux centraux pour le mouvement ${ }^{29}$. A partir de 2001, l'hostilité ambiante envers les musulmans est prégnante et c'est dans ce contexte que Fouad Imarraine, membre du CMF est invité à intervenir lors du 1er FSE dans un séminaire intitulé « La place de l'islam en Europe et l'islamophobie », organisé par la LDH et la FIDH.

23. Pour une description de tous ces groupes, voir Crettiez X., Sommier I. (dirs.), La France rebelle : tous les mouvements et acteurs de la contestation ( $2^{\mathrm{e}}$ ed.), Paris, Michalon, 2006.

24. Voir notamment: Gorce B., «Dialogue interreligieux. Des intellectuels musulmans censurés. Entretien. P. Gilles Couvreur. Secrétaire du Secrétariat pour les relations avec l'islam (SRI) », La Croix, 14 décembre 1995.

25. Tels le MRAP, les ASTI, la LDH et le CEDETIM.

26. Créée par la Ligue de l'enseignement et désormais gérée par la LDH et Le Monde diplomatique. http://www.islamlaicite.org/

27. Un des réseaux transnationaux défendant les causes des « sans ». http://www.novox.ras.eu.org/. En France, "No Vox » regroupe les associations comme le DAL, AC !, APEIS, le CDSL, IPAM, Droit paysan et Droits devant !!. Quelques militants avaient déjà travaillé avec le MIB sur les questions des sans-papiers et de la double peine.

28. Bové J., Retour de Palestine, Paris, Mille et une nuits, 2002.

29. Pleyers G., «Après le 11 septembre 2001. Entre mondialisation libérale et choc des civilisations », in Wieviorka M. (dir.), Un autre monde : contestations, dérives et surprises dans l'antimondialisation, Paris, Balland, 2003, pp. 195-213. 
Pour le second FSE, qui s'est tenu en région parisienne en 2003 des militants du CMF et du MIB ont été désignés membres du comité d'initiative français et du secrétariat d'organisation et ont pu ainsi influencer le choix des thèmes, séminaires et conférenciers lors des nombreuses «assemblées européennes de préparation ", obtenant finalement trois séances plénières et douze séminaires. Ce processus se répète l'année suivante avec le fait que, même s'ils ne font pas partie du comité organisateur, des membres du MIB et du CMF participent à l'organisation et prennent la parole pendant des séminaires à Londres. En juin 2003, le CMF unit officiellement ses forces avec celles du MIB et celles de groupes proches comme DiverCité 30 à Lyon, afin de former un réseau nommé « Résistances citoyennes» affilié aux « No Vox» 31 . Le 9 juin, ils organisent une table ronde préparatoire à Saint-Denis, en vue du FSE de la même année, intitulée « Quelles résistances citoyennes pour une justice globale ? ». La Confédération paysanne de José Bové invite le MIB et le CMF à participer à la manifestation du Larzac en 2003 où a été mis en place un village appelé « Résistances citoyennes» 32 . Cela inaugure officiellement leur participation dans le mouvement français et la relation avec José Bové est, depuis, demeurée forte. Quelques membres du MIB et du CMF ont même soutenu sa candidature aux élections présidentielles de 2007 en tant que membres du groupe «Banlieues et immigration avec José Bové » ${ }^{33}$.

\section{Le rôle de Tariq Ramadan}

Tariq Ramadan est sans conteste l'une des personnalités les plus influentes dans le développement de l'implication des musulmans et des associations musulmanes dans l'altermondialisme, particulièrement en France. L'accueil réservé à son action est également représentatif des opinions divergentes qui se côtoient au sein d'un même mouvement au sujet du rôle de la religion et, plus précisément celui de l'islam. A travers ses écrits, il encourage les musulmans d'Europe à être des citoyens actifs et insiste sur l'importance d'une implication sociale, d'une participation à la vie politique et à ce qu'il nomme la «résistance économique ${ }^{34}$ ». Il s'oppose à l'économie de marché et condamne les Etats du Golfe, surtout pour leur rôle dans ce système. En réalité, selon lui, il existe une opposition binaire entre les principes islamiques et le capitalisme :

30. Voir http://divercite.free.fr/

31. Pour plus d'informations sur la création de « Résistances citoyennes » et sur sa participation au FSE en 2003, voir Hmed C., « Aux marges de l'altermondialisme. La participation des classes populaires issues de l'immigration au $2^{\mathrm{e}}$ Forum social européen ", in Cadiou S., Dechezelles S., Roger A. (dirs.), Passer à l'action. Les mobilisations émergentes, Paris, L'Harmattan, 2007, pp. 249-269.

32. Du 8 au 10 août 2003, le plateau du Larzac a été le lieu d'une importante manifestation altermondialiste (appelée «Larzac 2003 »), répondant à l'appel de la Confédération paysanne et d'un vaste collectif d'associations et de syndicats. Plus de 200000 personnes se sont réunies pendant ces trois jours pour réfléchir aux impacts de la libéralisation du commerce sur leur vie quotidienne. Voir : http://www.larzac.org/resister/resistances.php

33. Coroller C., « Bové des champs essaime en banlieue. Enquête sur les réseaux du candidat antilibéral », Libération, 21 février 2007.

34. Ramadan T., Les Musulmans d'Occident et l'avenir de l'islam, Arles, Sindbad-Actes Sud, 2003. 
«Les enseignements islamiques sont intrinsèquement en opposition avec les fondements et la logique du système capitaliste néo-libéral et les musulmans qui vivent dans la tête du système ont la responsabilité supérieure de proposer, avec tous ceux qui travaillent dans le même sens, des solutions pour en sortir et permettre une économie plus juste et un commerce plus équitable 35 ».

Il est l'un des rares intellectuels musulmans - et certainement le seul personnage européen musulman important - à critiquer ainsi le capitalisme et à tenir un discours altermondialiste. On pourrait le qualifier de principal théoricien de la participation musulmane dans les mouvements pour la justice globale. Ses thèmes de prédilection ne concernent pas uniquement les problèmes économiques, mais aussi ceux habituellement liés aux préoccupations de nombreux musulmans, comme les événements se déroulant au Moyen-Orient.

Tariq Ramadan, présent à tous les FSE, est apparu pour la première fois en tant qu'orateur aux côtés de Fouad Imarraine à Florence. Au printemps 2003, il entre dans la sphère du mouvement, après avoir été reçu par une délégation de l'organisation altermondialiste ATTAC-France ${ }^{36}$. Le 4 juin 2003, il publie une tribune sur le site Internet oumma.com ${ }^{37}$. Dans cet article, il affirme que le mouvement altermondialiste ne prend pas suffisamment en compte la diversité culturelle et religieuse et que son discours reste "nourripar de vieux schémas coloniaux». Il invite ensuite le mouvement à entamer un dialogue avec «le monde de l'islam ». Le 9 juin, Ramadan participe à la table-ronde de Saint-Denis aux côtés de Bernard Cassen, président d'honneur d'ATTAC-France, qui répond à ses critiques en s'appuyant sur le fait que les militants n'ont pas besoin de réduire leur identité à des croyances religieuses ${ }^{38}$. Ceci marque le début d'une bataille intellectuelle entre les deux hommes, une confrontation ouvrant un débat au sein même d'ATTAC autour de la question de la laïcité et, spécifiquement, celle de l'importance à donner aux groupes musulmans depuis le début de la préparation du deuxième FSE. Pierre Khalfa, syndicaliste et membre du conseil scientifique d'ATTAC, plus ouvert au défi lancé par Ramadan, accepte l'idée que le mouvement doit faire davantage d'efforts pour intégrer les courants politiques inspirés de l'islam ${ }^{39}$. Ramadan a donc initié un débat impor-

35. Ramadan T. (dir.), Les Musulmans face à la mondialisation libérale : quelles résistances pour une justice globale?, Lyon, Tawhid, 2003, p. 17.

36. L'Association pour la taxation des transactions financières pour l'aide aux citoyennes et citoyens (ATTAC) est un réseau présent dans de nombreux pays et travaille sur des sujets divers: l'Organisation mondiale du commerce et les institutions financières internationales, la dette, la taxation des transactions financières, les paradis fiscaux, les services publics, l'eau, les zones de libres-échanges (Méditerranée, Amérique, Europe...). Voir : http://www.attac.org/

37. On trouvera une version plus étoffée de cette tribune dans l'hebdomadaire Politis: Ramadan T., «Les défis du pluralisme et les contradictions du monde musulman », Politis, n ${ }^{\circ} 756,19$ juin 2003.

38. Cassen B., "Altermondialisation et islam », Politis, n759, 10 juillet 2003.

39. Khalfa P., «Islam et altermondialisme : le défi de l'universel », Politis, ${ }^{\circ} 766,11$ septembre 2003. Voir aussi « L'islam, l'enjeu de l'intégration », Libération, 11 novembre 2004. 
tant entre les militants français au sujet de l'islam et du rôle des musulmans dans ce mouvement, mais celui-ci va dégénérer après ce que l'on nomme communément « l'affaire Ramadan ». Son article au sujet des « intellectuels communautaires », publié le 3 octobre 2003 sur le site oumma.com puis sur une liste de discussion par Internet préparant le FSE de novembre, causa des remous si importants que ceux-ci en sont venus à occulter la totalité du FSE à Paris ${ }^{40}$.

Ramadan est de nouveau invité au FSE l'année suivante à Londres; il est même programmé en tant qu'orateur dans huit séminaires différents, bien que certains d'entre eux aient lieu simultanément. A ce moment, il est inconnu au Royaume-Uni et, malgré quelques oppositions de la National Union of Students (NUS), sa venue se déroule sans incident et la presse s'intéresse très peu à sa participation ${ }^{41}$. Néanmoins, en France, on continue à débattre sur sa participation au forum, le président de SOS Racisme dénonçant sa venue ainsi que celle d'autres "islamistes intégristes 42 ». Quelques jours après la fin du FSE, un ouvrage critiquant Ramadan, et en particulier sa tentative d'entrée dans le mouvement altermondialiste, est publié ${ }^{43}$. Deux mois après, une édition de Politis ${ }^{4}$ titrait «Tariq Ramadan : islamiste ou citoyen? ». Durant le 4e FSE à Athènes en 2006, Ramadan est à nouveau présent et il prend la parole lors d'un séminaire sur l'islamophobie et la controverse des caricatures danoises. Une nouvelle fois, il entre en conflit avec des groupes féministes comme Femmes solidaires ${ }^{45}$ qui décide "de ne pas participer aux travaux de ce forum comme il était préou» et se retrouve face à son adversaire, la journaliste Caroline Fourest, qui continue sa lutte contre la «complaisance » du mouvement altermondialiste à son égard ${ }^{46}$.

\section{Des identités tolérantes?}

Le résultat le plus évident de la mobilisation des musulmans et de l'implication des associations musulmanes et autres organisations « issues de l'immigration » dans la galaxie altermondialiste est l'ensemble des débats, désaccords, réper-

40. Ramadan a publié un texte «Critique des (nouveaux) intellectuels communautaires » que beaucoup définissent comme antisémite, voir : http://oumma.com/Critique-des-nouveaux et Voir : http://www.politis.fr/article716.html. Moins de deux jours avant l'ouverture du second FSE, des féministes publièrent aussi un appel condamnant sa participation : "Ramadan antiféministe ", Libération, 12 novembre 2003. Le MFPF et le CADAC faisaient partie des groupes signataires de ce texte. Sur les divisions sur ces questions au sein des mouvements féministes français, voir Dot-Pouillard N., «Les recompositions politiques du mouvement féministe français au regard du hijab » SociologieS, premiers textes, mis en ligne le 31 octobre 2007, http://sociologies.revues.org/document246.html

41. Le seul article paru dans la presse nationale a dressé de lui un portrait plutôt positif. Bunting M,.

"Muslims urged to embrace their role in the West", The Guardian, 16 octobre 2004.

42. Sopo D., "Un “autre monde” mais avec qui ? ", Libération, 14 octobre 2004.

43. Fourest C., Frère Tariq : discours, stratégie et méthode de Tariq Ramadan, Paris, Grasset, 2004.

44. Politis, $\mathrm{n}^{\circ} 835,20$ janvier 2005.

45. Voir: http://www.femmes-solidaires.org/

46. «Des féministes contre la présence de Ramadan », L'Humanité, 9 mai 2006. 
cussions et scissions que celles-ci ont entraîné au sein de ces groupes et associations altermondialistes dans les deux pays. Selon Donatella Della Porta, les «identités tolérantes 47 » sont l'un des traits principaux des forums sociaux et du mouvement altermondialiste, soit la capacité des personnes impliquées à inclure de nombreux groupes différents au nom d'un front uni contre le néo-libéralisme. La participation musulmane montre qu'il existe des limites à cette tolérance et que ces mouvements ont peut-être fait face à leur plus grand défi en intégrant des associations musulmanes. Des tensions similaires existaient au sein de ces associations qui cherchaient à joindre ces mouvements. De plus, en France, de nombreuses associations situées dans les « quartiers populaires » ont maintenu une attitude critique envers les altermondialistes. A ces tensions internes s'ajoutent les opinions des commentateurs extérieurs, en particulier l'attitude critique de certains journalistes et universitaires vis-à-vis de ces nouveaux développements (voir infra).

\section{Altermondialistes (et musulmans) divisés}

Le premier point de discorde au sein des mouvements altermondialistes est le fait que les « laïcs » souvent défendent l'idée d'une sphère publique libérée de toute religion mais, bien entendu, seuls les musulmans et leur religiosité sont vraiment visés. Comme on pouvait s'y attendre, cette vision a trouvé un grand écho en France, mais aussi par certains éléments de la gauche radicale britannique ${ }^{48}$. Certains se sont très certainement montrés réticents à l'idée de faire des concessions en faveur de la participation musulmane comme le fait, par exemple, d'ouvrir des espaces dédiés à la prière. En Grande-Bretagne, la participation du groupe JP au mouvement anti-guerre, le premier groupe musulman visible, n'a pas créé de véritables problèmes car ses membres ont cherché à travailler activement avec d'autres groupes et à discuter de thèmes auxquels les participants traditionnels à ce mouvement étaient liés, comme la justice et l'égalité. Il est d'ailleurs important de souligner que les débats les plus houleux tournent autour de la question de la participation d'associations et leurs représentants, soupçonnés de promouvoir une certaine forme d'Islam politique. Ce problème a éclaté au grand jour lorsque la MAB est devenue un partenaire officiel de la SWC. Le groupe est proche des Frères musulmans et, par conséquent, certains se sont mis à redouter d'intégrer un groupe réactionnaire, hostile à la démocratie, aux homosexuels et aux droits des femmes ${ }^{49}$. Les dirigeants de la coalition ont, dès le

47. Della Porta D., "Multiple belongings, tolerant identities, and the construction of 'another politics': between the European Social Forum and the local social fora”, in Della Porta D., Tarrow S. (eds.), Transnational Protest and Global Activism, Lanham, Rowman and Littlefield, 2005, pp. 175-202. Ces «identités tolérantes » seraient caractérisées par l'inclusion et le soulignement positif de la diversité.

48. Ce fut, par exemple, le cas de l'Alliance for Workers Liberty (AWL) qui essaya de bannir la $\mathrm{MAB}$ du mouvement anti-guerre.

49. Certains militants de la CND ont quitté la SWC en raison de la participation de la MAB, même si en réalité, on peut douter du fait que les membres de cette dernière faisaient partie de la base de la participation musulmane. 
début, tenté d'apaiser cette peur, mais la controverse a repris de plus belle avec la découverte de la nature du journal distribué par leurs membres à l'occasion d'un cortège anti-guerre en septembre 2002. Celui-ci contenait un article portant sur les droits de l'Homme dans l'islam et décrivait la justification pour la peine de mort pour l'apostasie ${ }^{50}$. Cela peut paraître "dérisoire » comparé au scandale provoqué par Youssef al-Qaradâwî lorsqu'il est invité par Ken Livingstone à parler à la conférence « Hijab » à Londres en 2004 et au FSE. Le cheikh qatari d'origine égyptienne, qui dirige le Conseil européen de la fatwa et de la recherche, a suscité de nombreuses polémiques à cause de ses prises de positions sur des thèmes comme les attentats-suicides, les homosexuels et la loi française contre le port de signes religieux ostensibles. Sa visite a entraîné de nombreuses plaintes, notamment par les féministes, les groupes « Lesbien, gay, bi-sexuel, transsexuel » (LGBT) et les représentants des communautés hindoue et sikh ${ }^{51}$.

En France, les débats internes au mouvement altermondialiste ne se focalisent pas sur des associations particulières, mais plutôt sur une personnalité particulière : Tariq Ramadan. Au fond, beaucoup ne sont pas convaincus de ses motifs et pensent qu'il ne s'est pas complètement débarrassé de l'héritage de son grandpère Hassan al-Banna, fondateur des Frères musulmans en Egypte. Un des organisateurs du FSE de Paris affirme par exemple qu'il est « quelqu'un qui vient de l'intégrisme le plus traditionnel et qui est en train d'en sortir avec beaucoup de difficulté $52 »$. Le fait qu'il parle d'une perspective islamique est tout autant inadmissible pour d'autres et vu comme un exemple du "communautarisme musulman 53 ». En d'autres termes, privilégier les valeurs de l'islam aux valeurs universelles et/ou supposément républicaines. Ramadan essaye sans trop de succès de retourner la critique et de l'utiliser contre d'autres dans son désormais célèbre article sur les « intellectuels communautaires ».

En France, lors de la préparation du deuxième FSE, de nombreux altermondialistes se sont montrés réticents à l'idée de travailler avec des associations musulmanes. La présence d'une femme musulmane portant le voile en a ainsi inquiété plus d'un, comme l'explique Siham Andalouci, membre du Bureau national du CMF : «la première fois que je suis entrée dans une pièce, lors d'une réunion préparatoire du FSE, l'oratrice s'est arrêtée de parler, tellement elle était surprise de voir une fille voilée 54 ». De nombreux militants

50. Voir Tamimmi A., "Islam and Human Rights", Inspire, 28 septembre 2002.

51. Certains représentants de ces groupes formèrent une coalition temporaire, la London Community Coalition pour s'opposer à ces visites. Au final, al-Qaradawi ne participa pas au FSE.

52. Freyssenet E., Validire J-L., Portes T., « Le débat sur la place de l'islam dans l'altermondialisme divise les participants ", Le Figaro, 14 novembre 2003.

53. Ce concept est souvent utilisé telle une accusation par les élites politiques ; même si elles encouragent en réalité un tel phénomène à travers leur manière de diriger et leur tentative de gérer la « question musulmane ». Voir Geisser V., Zemouri A., Marianne et Allah. Les politiques français face à la question musulmane, Paris, La Découverte, 2007.

54. Askolovitch C., « L’encombrant M. Ramadan », Le Nouvel Observateur, n²031, 9 octobre 2003. 
altermondialistes ont affirmé qu'ils n'étaient pas habitués à travailler avec des individus et des associations religieuses. Néanmoins, cette position peut sembler hypocrite, dans le mesure où la participation d'autres associations religieuses comme le Secours catholique ne semblait pas problématique ${ }^{55}$. Les membres d'ATTAC sont divisés sur la manière d'interpréter le principe de laïcité dans ce contexte. Certains s'accrochent à une interprétation dans laquelle les croyances religieuses personnelles doivent rester de l'ordre de la sphère personnelle. Pour Bernard Cassen par exemple, le mouvement peut inclure des débats et des discussions avec des musulmans, mais seulement en tant qu'individus, et non «en tant que musulmans»56. Effectivement, comme le souligne Valérie Amiraux, "c'est moins le principe de laïcité qui rend l'expression publique de la religion difficile en France que la perception que les gens ont de son sens 57 ».

Par ailleurs, des débats au sein des communautés musulmanes et leurs associations concernant leur participation aux campagnes altermondialistes n'ont pas été moins rudes, faisant apparaître un nombre important de dissensions, y compris au sein de la MAB. Certains membres de l'association se montrent réticents à l'idée de s'allier avec la SWC, d'opter pour le militantisme de rue et la confrontation directe avec l'Etat.

En réalité, les leaders tiennent à souligner qu'ils ne sont pas d'accord sur tout et que leur alliance avec la gauche n'est qu'un « mariage de convenance »:

«Il n'y a pas d'alliance permanente. Ce sont des alliances fondées sur des problèmes pour lesquelles nous trouvons une base commune avec la gauche, même avec l'extrême gauche, comme la politique étrangère, la Palestine et l'Irak. Nous ne partageons pas le même point de vue sur beaucoup d'autres problèmes ${ }^{58}$. »

Il y avait aussi quelques tensions à l'intérieur du MIB sur le fait de travailler avec des groupes confessionnels comme le CMF. Leurs militants et certains autres de Résistances citoyennes sont extrêmement frustrées par la réticence de nombreux autres acteurs, plus établis en France, à travailler avec eux et à inscrire leurs demandes dans le «mouvement social». Yamin Makri du CMF accuse les leaders de ce mouvement en France d'avoir tout simplement un problème avec l'islam :

55. Voir le témoignage d'un militant du CMF dans Hmed C., " Aux marges de l'altermondialisme ", p. 264.

56. Cassen B., « Ces altermondialistes en perte de repères », Politis, n 835,20 janvier 2005.

57. Amiraux V., "Speaking as a Muslim. Avoiding religion in the French public space", in Jonker G., Amiraux V. (eds.), Politics of Visibility. Young Muslims in European Public Spaces, Bielefeld, Transcript, 2006, p. 34.

58. Vasagar J., "Threat of war: meeting of minds", The Guardian, 14 février 2003. 
«Il y a une nouvelle réalité militante sociologique et beaucoup de cadres ont du mal à accepter que, parmi les mouvements de résistance, certains s'inspirent de l'islam : il serait tellement plus simple si ces musulmans pouvaient faire abstraction de leur religiosité ou rester entre eux ${ }^{59}$.»

Effectivement, les résistances qu'ils ont rencontrées les ont forcés à adopter des positions critiques envers ces acteurs et, lors du deuxième FSE, ces groupes ont passé paradoxalement presque plus de temps à dénoncer les altermondialistes «traditionnels » qu'à critiquer la mondialisation économique et ses supposés effets sur ceux qu’ils représentent dans les banlieues.

\section{Les réactions journalistiques}

Les observateurs extérieurs critiquant les alliances qui ont vu le jour entre les altermondialistes et les associations musulmanes, en particulier en France, ne manquaient pas. En Grande-Bretagne, le plus important de ces détracteurs est Nick Cohen, journaliste de gauche qui avait soutenu l'invasion de l'Irak en 2003. Celui-ci dénonce l'implication de la MAB, qu'il décrit comme un mouvement d'extrême droite, disqualifie et blâme le mouvement anti-guerre "parce qu'il n'a pas pu s'opposer à la contre-révolution lorsque la guerre a été terminée 60 ». Pour illustrer ses propos, il fait remarquer que, lors du FSE de 2004, la majorité des orateurs ont soutenu la « résistance irakienne » et que l'on a refusé de donner la parole à Subhi al Mashadani, secrétaire de la Fédération irakienne des syndicats, parce que celui-ci était accusé par des éléments perturbateurs de « collaboration » avec les Américains. Caroline Fourest s'est également montrée très critique envers le FSE et plus particulièrement vis-à-vis des invitations faites à Tariq Ramadan, en jugeant très sévèrement ce qu'elle considère comme de "l'aveuglement à gauche envers l'islamisme 61 ». Selon elle, la présence de Ramadan au sein de ce mouvement est tout simplement une "rampe de lancement au service $d u$ renouveau islamique 62 ». Le journal satirique Charlie Hebdo, lui, titrait l'un de ses articles: «Un autre jihad est possible » et d'autres médias de gauche, comme L'Humanité ou Le Nouvel Observateur, ont eux aussi durement critiqué son engagement ${ }^{63}$. Dans le monde universitaire, Pierre-André Taguieff, une des cibles de la diatribe

59. «Les associations musulmanes et organisations issues de l'immigration dans le mouvement altermondialiste en France », in Ramadan T. (dir.), Les Musulmans face à la mondialisation libérale, p. 67. Entretien publié pour la première fois dans la lettre d'information électronique d'ATTAC France, Grain de Sable, $\mathrm{n}^{\circ} 437$.

60. Cohen N., What's Left?: How Liberals Lost Their Way, London, Fourth Estate, 2007, p. 288.

61. Fourest C., La Tentation obscurantiste, Paris, Grasset, 2005, p. 29.

62. Ibid., p. 125.

63. Venner F. "FSE: un autre jihad est possible», Charlie Hebdo, 29 septembre 2004. Askolovitch C., "Les gauchistes d'Allah », Le Nouvel Observateur, n²085, 21 octobre 2004. Piérot J.-P., «Les deux faces du FSE », L'Humanité, 23 octobre 2004. Le débat ne s'est pas arrêté là, dans l'édition du 21 décembre 2004 de Libération, on pouvait lire un dossier intitulé «Polémique à gauche sur l'intégration des islamistes ». 
de Ramadan contre les «intellectuels communautaires », répond par le biais d'un chapitre de son livre qui alerte sur les dangers de «l'islamisme et du néo-gauchisme ${ }^{64}$ ».

\section{Les résultats de la participation : de nouvelles campagnes, mouve- ments et partis politiques}

L'effet le plus notable de la mobilisation des musulmans en GrandeBretagne est la formation d'un nouveau parti politique Respect - the Unity Coalition ${ }^{65}$. Ce parti est désormais considéré comme un modèle à suivre par certains militants musulmans en France car aucun mouvement politique de ce type n'a encore vu le jour en France

\section{Respect}

Cette initiative, directement issue du succès du mouvement anti-guerre, est dirigée entre autres par l'ancien député du parti travailliste George Galloway. Ce parti veut fédérer tous ceux situés à la gauche dudit parti. Il est donc le successeur de la Socialist Alliance qui n'avait jusqu'alors pas rencontré le succès attendu. Sa stratégie électorale, néanmoins, est essentiellement fondée sur un ciblage des électeurs musulmans désabusés et déçus par la décision du gouvernement d'envahir l'Irak. La plupart des personnages clés impliqués dans la SWC ont continué à jouer un rôle essentiel au sein de Respect, qui a officiellement été formé en janvier 2004. Comme on pouvait s'y attendre, Respect a largement été représenté lors de la troisième édition du FSE à Londres, et notamment par une grande réunion «off ». Le succès du parti est remarquable au niveau local, dans des circonscriptions constituées d'un grand nombre d'habitants musulmans, avec actuellement environ vingt conseillers municipaux élus. Son plus grand succès à ce jour est ce qui s'est passé lors des élections législatives de 2005, quand Galloway a été élu membre du Parlement pour la circonscription de Bethnal Green and Bow. Non seulement c'est la première fois qu'un parti situé à gauche du parti travailliste remporte un siège à la Chambre des communes depuis 1951, mais c'est aussi le seul petit parti à remporter un siège dans les circonscriptions anglaises. Malgré ce succès, le parti doit faire face à de nombreuses critiques. Sa

64. Taguieff P.-A., Prêcheurs de haine : traversée de la judéophobie planétaire, Paris, Mille et une nuits, 2004. Voir l'épilogue intitulé « Nouvelle judéophobie, islamisme et nouvel anti-capitalisme: dangereuses convergences », pp. 819-945.

65. «Respect» est l'acronyme de Respect, Equality, Socialism, Peace, Environmentalism, Community et Trade Unionism. Voir http://www.respectcoalition.org/

66. Un tel terme est rarement utilisé dans le discours politique britannique. Il est intéressant en ce qu'il fait partie de ce mouvement général vers la critique du multiculturalisme. Nick Cohen compare Respect au British National Party (BNP) tout comme l'a fait Nick Ryan. Voir "Alien nations”, The Guardian, 10 décembre 2006. George Galloway défendait le parti face aux accusations en affirmant que "la Respect Coalition n'est pas engagée dans un appel communautaire au vote musulman. Nous reconnaissons que de nombreux musulmans ne peuvent se convaincre de voter pour le parti travailliste. Nous faisons campagne pour gagner leur vote 
stratégie électorale a été qualifiée de «communautariste 66 » parce qu'elle courtise explicitement les musulmans. On a également reproché au parti le fait que certains de ses conseillers entretiennent des relations avec des associations musulmanes liées à Jamaat-e-Islami (JI) même si, au Bangladesh, ses militants s'opposent de façon virulente aux militants de gauche ${ }^{67}$. Un autre problème réside dans l'équilibre entre les groupes LGBT et les inquiétudes féministes par rapport aux musulmans les plus conservateurs au sein du parti. Respect est actuellement sujet à une crise majeure et s'est scindé en deux depuis fin 2007, bien que cela n'ait rien à voir avec les problèmes susmentionnés ${ }^{68}$.

Les avis au sein de la MAB divergent aussi clairement sur la question de leur rôle dans le mouvement anti-guerre. Certains membres croient que leur alliance avec la SWC, malgré une certaine amélioration de leur visibilité, arrive à son terme. Fin 2005, une lutte pour le pouvoir s'engage au sein de l'association et une partie de ceux qui étaient impliqués dans le mouvement antiguerre, comme Azam Tamimi, Anas Al Tikriti et Soumaya Ghannaoushi sont forcés de prendre leurs distances avec la direction. Ils poursuivent la création de la British Muslim Initiative (BMI) au début 2006, qui continue à travailler avec la SWC pour l'organisation de manifestations. Naturellement, ceci n'est pas sans effet, car la BMI ne peut mobiliser autant de ressources que la MAB. De la même manière, les groupes qui se sont formés en Grande-Bretagne ont été démantelés à l'instar du JP, de l'IMAN ou encore de la LMC. En réalité, on reconnaît aujourd'hui que la présence musulmane au troisième FSE a été superficielle, et que la majorité des musulmans qui se sont mobilisés l'ont fait individuellement, sans rejoindre aucun groupe. Il apparaît également que les coalitions et les liens entre les militants musulmans et non-musulmans ont été difficiles à maintenir. Les spécialistes du mouvement anti-guerre affirment que "l'interaction entre les musulmans et non-musulmans était partielle, superficielle, par moment uniquement symbolique et par-dessus tout temporaire 69 ". Ceci contraste avec les jugements enthousiastes et optimistes de certains leaders quant à l'apparition d'une nouvelle forme de "radicalisme politique islamique » en Grande-Bretagne, soulignant le succès des coalitions progressistes réalisant la symbiose des musulmans avec les autres militants 70 .

pour une alternative de gauche au parti travailliste », The Guardian, 7 juin 2004.

67. Husain E., The Islamist: Why I Joined Radical Islam in Britain, What I Saw Inside and Why I Left, London, Penguin books, 2007, p. 281. JI (" parti islamique ») est un parti islamiste au Bangladesh, insipré du même parti au Pakistan fondé par Sayyid Abul Ala Maududi.

68. La scission oppose essentiellement le SWP et d'autres membres fondateurs de Respect comme Galloway et Yaqoob. Ces derniers ont créé une faction séparatiste nommée "Respect renewal", voir http://www.respectrenewal.org

69. Pickerill J., Webster F., Gillan K. "Muslim anti-war activism as positive political engagement", Chatham House Briefing Paper. Islam, Politics and Security in the UK, 2007, http://www.chathamhouse.org.uk/publications/papers/download/-/id/546/file/10487_bp1007islamuk.pdf

70. Yaqoob S., "British Islamic political radicalism”, in Abbas T. (ed.), Islamic Political Radicalism: a European Perspective, Edinburgh, Edinburgh University Press, 2007, pp. 279-294. 
Une des premières conséquences de l'implication des « associations des banlieues »dans le processus du FSE en France est l'intensification de leur collaboration avec certains membres de la gauche radicale. La création en mars 2004 d'un collectif opposé à l'interdiction du voile («Une école pour tous-tes ») en est le premier et le plus flagrant des exemples. Les liens tissés avec des personnalités comme la féministe Christine Delphy ont amené plus tard à des collaborations entre associations de banlieues et la gauche radicale lors du FSE en 2004 comme le séminaire controversé sur le Hijab. A la suite de cette campagne, le mouvement des «Indigènes de la République » est lancé en 2005, en regroupant nombre de ces mêmes acteurs. Néanmoins, la grande majorité des acteurs altermondialistes n’ont pas apporté leur soutien de telles campagnes et les efforts de ceux qui ont souhaité intégrer des groupes musulmans dans le « mouvement social » ont majoritairement échoué. Les groupes eux-mêmes ont été restreints par manque de ressources financières, d'où leur absence du dernier FSE à Athènes en 2006. Pour pallier à ces difficultés, ils ont adapté le format du forum : du 22 au 24 juin 2007, la ville de Saint-Denis accueillait le premier Forum social des quartiers populaires (FSQP). Ceci constitue leur réponse au mouvement altermondialiste que beaucoup continuent de qualifier de «parlotte pour intellectuels» ou pour «bobos » qui font le tour du monde ("les globe-trotters de la militance ») sans jamais vraiment salir leurs mains et laissant les problèmes au pas de leur porte ${ }^{71}$. L'un des objectifs du FSQP est d'être l'instigateur d'un mouvement politique qui représenterait les inquiétudes des militants, un «mouvement politique autonome ». Le besoin d' " autonomie » et donc la réticence à joindre leurs forces à celles de partis politiques de gauche bien établis sont le résultat d'une suspicion envers ces acteurs profondément ancrée chez un grand nombre de groupes. L'expérience de la «Marche des beurs 72 » et sa "récupération » par le parti socialiste est restée dans la mémoire de nombreux activistes. En effet, il existe un grand manque de confiance envers toute la classe politique ("nous ne déléguerons nos luttes et nos causes à personne, ni aux partis de droite, ni aux partis de gauche, qui font de la récupération, du clientélisme une politique pour nos quartiers ${ }^{73}$. ")

Dans leurs recherches sur la mobilisation politique autour des problèmes

71. Entretien avec Abdelaziz Chaambi (militant du CMF), janvier 2008.

72. La marche pour l'égalité et contre le racisme, partie de Marseille le 15 octobre 1983, s'achève à Paris le 3 décembre par un défilé réunissant 100000 personnes.

73. Communiqué du MIB, «Pour un mouvement politique autonome des quartiers », 23 mars 2006 : http://www.saphirnews.com/Pour-un-mouvement-politique-autonome-desquartiers_a2563.html 
des relations ethniques et de l'immigration en Europe, Ruud Koopmans, Paul Statham, Marco Giugni et Florence Passy affirment que les variations selon les différents pays sont le résultat de conceptions différentes de l'identité nationale et leur cristalisation dans les spécificités politiques nationales de l'intégration et de la citoyenneté. Ainsi, ces notions agissent comme des «structures d'opportunités discursives » pour les acteurs qui s'y mobilisent ${ }^{74}$. Examiner la participation des musulmans dans les mouvements altermondialistes nous permet de voir comment ces «structures ", dans chaque société, ont affecté ces processus. En Grande-Bretagne, les organisateurs se sont donnés beaucoup de mal pour s'adapter aux besoins des participants musulmans. Il y a même eu un appel à la prière pour les participants musulmans lors de certaines manifestations organisées par la SWC. De telles initiatives seraient inconcevables dans le contexte français où le fait même de porter un foulard est problématique pour beaucoup. La société française reste suspicieuse, sinon hostile, envers la religion portée dans la sphère publique, surtout envers l'islam. Néanmoins, le principe de laïcité, que l'on évoque souvent pour justifier cette hostilité, est souvent employé de la même façon par les associations musulmanes qui sont accusées de le violer. Comme le fait remarquer Armando Salvatore, dans les controverses impliquant des musulmans en France, ces derniers ont euxmêmes tendance à affirmer leur allégeance aux valeurs läques et contre toute forme de «communautarisme 75 ». Comme nous l'avons vu, la notion de laïcité est contestée et l'exemple des altermondialistes illustre le fait que certains la perçoivent comme une garantie pour la liberté de pratiques religieuses, alors que d'autres pensent que cela implique la nécessité de la confiner à la sphère privée. Par conséquent, des deux côtés, le terme est utilisé comme un outil discursif pour légitimer une position. Un leader de la CMF par exemple explique comment sa vision de ce concept a changé au fil du temps :

«Avant, pour nous, c'était un synonyme d'anti-religion, de mécréance ou de guerre contre les religions, aujourd'hui on se réclame laic, la laïcité c'est un modèle... C'est la neutralité de l'Etat, c'est la liberté de conscience et la liberté de culte. Tu peux croire en qui tu veux tant que tu ne me l'imposes pas, c'est ça le cadre laïc... La manière dont le CFCM [Conseil Français du Culte Musulman] a été mis en place est une atteinte à la laïcité ! 76 »

L'introduction de revendications basées sur l'identité musulmane a profondément marqué le mouvement altermondialiste mais cela a été géré de deux manières complètement différentes en Grande-Bretagne et en France. Les lea-

74. Koopmans R., Statham P., Giugni M., Passy F., Contested Citizenship: Immigration and Cultural Diversity in Europe, Minneapolis, University of Minnesota Press, 2005.

75. Salvatore A., "Authority in question: secularity, republicanism and 'communitarianism' in the emerging Euro-Islamic public sphere”, Theory, Culture E Society, mars 2007, n²4, pp. 135-160.

76. Entretien avec Abdelaziz Chaambi, op. cit. 
ders britanniques ont tenu à tirer profit de cette nouveauté bien que la nature superficielle de ces alliances ait exprimé le fait qu'elles n'aient pas pu être maintenues. C'est aussi un reflet de la nature et de la faiblesse de la mouvance altermondialiste en Grande-Bretagne comparé à la France. Malgré la présence de nombreuses associations au FSE de 2004, la participation des militants musulmans a été moins importante qu'elle ne l'avait été à Paris un an auparavant, malgré le fait que les leaders français soient beaucoup plus hésitants à intégrer ces associations. Les connotations négatives associées au concept de communautarisme ont expliqué leur réticence à adopter l'approche pragmatique de leurs homologues outre-Manche. La réaction négative en France peut sembler surprenante si l'on considère que les militants des banlieues n'ont pas essayé de mobiliser systématiquement autour d'une identité islamique et que les associations impliquées pourraient être considérées comme beaucoup plus progressistes en France qu'au Royaume-Uni. Etant donné que ces acteurs en France ont des années d'expérience militante et que leur implication avec les altermondialistes ne constituait qu'une étape supplémentaire dans leur long parcours depuis la «Marche des beurs », on ne peut s'étonner du fait que ces groupes restent établis en France, tandis qu'en Grande-Bretagne ceux-ci se sont dissous. La mobilisation des musulmans britanniques, autour de la question irakienne en particulier, est une réussite sans précédent pour les altermondialistes britanniques. Pourtant, l'interaction entre le mouvement et les musulmans demeure très faible et le seul héritage de cette réussite est le parti Respect, actuellement en crise. Ce succès fugace illustre les difficultés et paradoxes liés à l'implication des musulmans et des associations qui les représentent au sein de l'altermondialisme européen.

\section{Liste des sigles}

AWL : Alliance for Workers, Liberty, http://www.workersliberty.org/ BMI : British Muslim Initiative, http://www.bminitiative.net/

BNP : British National Party, http://www.bnp.org.uk/ CAMPACC : Campaign Against Criminalising Communities, http://www.campacc.org.uk/

CND : Campaign for Nuclear Disarmament, http://www.cnduk.org/

CMF : Collectif des musulmans de France, http://www.lecmf.fr/

FOSIS : Federation of Student Islamic Societies, http://www.fosis.org.uk/ FSE : Forum social européen, http://www.fse-esf.org/ FSQP : Forum social des quartiers populaires, http://fsqp.free.fr/ GLA : Greater London Authority, http://www.london.gov.uk/ IMAN : International Muslim Activist Network ISB : Islamic Society of Britain, http://www.isb.org.uk/

JI : Jamaat-e-Islami JP : JustPeace, http://www.4justpeace.com/ * LMC : London Muslim Coalition 
MAB : Muslim Association of Britain, http://www.mabonline.net/

MCB : Muslim Council of Britain, http://www.mcb.org.uk/

MIB : Mouvement de l'immigration et des banlieues, http://mibmib.free.fr/

MP : Muslim Parliament, http://www.muslimparliament.org.uk/

NAAR : National Assembly Against Racism, http://www.naar.org.uk/

NMP : Newham Monitoring Project, http://www.nmp.org.uk/

NUS : National Union of Students, http://www.nusonline.co.uk/

SWC : Stop the War Coalition, http://www.stopwar.org.uk/

SWP : Socialist Workers Party, http://www.swp.org.uk/

* Ce site fonctionne plus. 